\title{
An Assistive Mobile Platform for Delivering Knowledge of Performance Feedback
}

\author{
Davide Neves \\ Universidade da Madeira, \\ Funchal, Portugal \\ davideneves@gmail.com
}

\author{
Athanasios Vourvopoulos \\ Madeira-ITI, \\ Universidade da Madeira, \\ Funchal, Portugal \\ athanasios.vourvopoulos@ \\ m-iti.org
}

Mónica Cameirão

Madeira-ITI,

Universidade da Madeira,

Funchal, Portugal

monica.cameirao@m-iti.org

\author{
Sergi Bermudez i Badia \\ Madeira-ITI, \\ Universidade da Madeira, \\ Funchal, Portugal \\ sergi.bermudez@m-iti.org
}

\begin{abstract}
Upper limb motor deficits caused by stroke have a big impact on a person's daily activities and independence. One strategy for promoting motor relearning consists on the delivery of meaningful feedback during rehabilitative training. In this paper we describe the development and first evaluation of a system that combines a portable arm orthosis device and a mobile application running on a tablet in order to provide knowledge of performance to stroke patients while performing therapy. Here we present preliminary results and discuss the potential of this technology.
\end{abstract}

\section{Categories and Subject Descriptors}

J.3 [Computer Applications]: Life and Medical Sciences health; K.4.2 [Computers and Society]: Social Issues - assistive technologies for persons with disabilities

\section{General Terms}

Human Factors

\section{Keywords}

Stroke, motor rehabilitation, knowledge of performance, mobile devices

\section{INTRODUCTION}

Motor impairment of the upper limb, cognitive and emotional sequels are commonly observed in stroke survivors $[1,2]$. These deficits have a huge impact on person's activities of daily living (ADL), making them dependent on others in order to do simple daily tasks. Rehabilitation is essential for motor learning and helping in the acquisition of skills that can improve independence in every day tasks. Here, several elements can contribute to a more successful rehabilitation process, resulting in the enhancement of the motor performance of the patient $[3,4]$. One important aspect in rehabilitation is to be able to provide meaningful and valuable feedback in order to help patients in their motor relearning tasks. Nowadays it is widely accepted that there are two types of extrinsic feedback that play a crucial role in providing information about the motor task execution, knowledge of results (KR) and knowledge of performance (KP) [5]. KR feedback is given after completing the desired task and relates to how well the task has been performed, while KP provides information about

Permission to make digital or hard copies of all or part of this work for personal or classroom use is granted without fee provided that copies are not made or distributed for profit or commercial advantage and that copies bear this notice and the full citation on the first page. To copy otherwise, to republish, to post on servers or to redistribute to lists, requires prior specific permission and/or a fee. REHAB 2014, May 20-23, Oldenburg, Germany Copyright $\odot 2014$ ICST 978-1-63190-011-2 DOI 10.4108/icst.pervasivehealth.2014.255278 what is being done during the execution of the training task in order to aid the patient in achieving the best outcome [5].

The benefits of the advances in technologies in the rehabilitation area are well known, and the applications range from brain computer interfaces [6] to robotic systems [7]. For instance, current computer based approaches for motor rehabilitation are very well suited to provide KR by embedding training in the form of games that provide quantitative measurements of results, which are displayed to the user $[8,9]$. However, KP is generally provided verbally by trained therapists during the task execution itself. To be able to provide meaningful KP requires the deployment of specific sensing technology capable of measuring and assisting the rehabilitation process in a safe and unobtrusive way, such as portable sensing devices. Combined with software applications that provide KP, these technologies can assist patients with motor deficits while allowing the capture of important physiological and kinematic information such as electro-my ographic signals or arm position.

The goal of this project is to develop a fully portable system that captures and processes multimodal information in order to provide KP. To accomplish this, we developed a mobile application for Android devices that pairs with a portable robotic arm device that has position and EMG sensing capabilities and assists the patient during movement execution. The mobile application is designed to provide real-time feedback on KP during task execution from both EMG and kinematic data. Subsequently, we did a pilot evaluation to assess the impact depending on the nature of the information used for KP: 1) physiologically based feedback where EMG signals are used to inform the patient about muscular activation, or 2) kinematic based feedback to provide the patient with elbow position and speed of movement.

\section{METHODS}

\subsection{Myo-electric limb orthosis}

We used the mPower 1000 (mpower1000, Myomo Inc., Boston, USA), a robotic limb orthosis that is portable and has one actuated degree of freedom (elbow join). The orthosis uses two electrode sensors placed on the biceps and triceps of the patient's paretic arm, thus reading its myo-electrical (EMG) signals plus the motor position (i.e. elbow flexion). By activating the biceps or triceps for arm flexion or extension, the EMG readings enable the device to assist the patient by driving its electric motor in order to support the desired movement.

\subsection{Mobile application}

The application is designed for mobile devices running Android OS (Google Inc., Mountain View, California, U.S.) to leverage the information gathered from the myo-electric orthosis to provide 

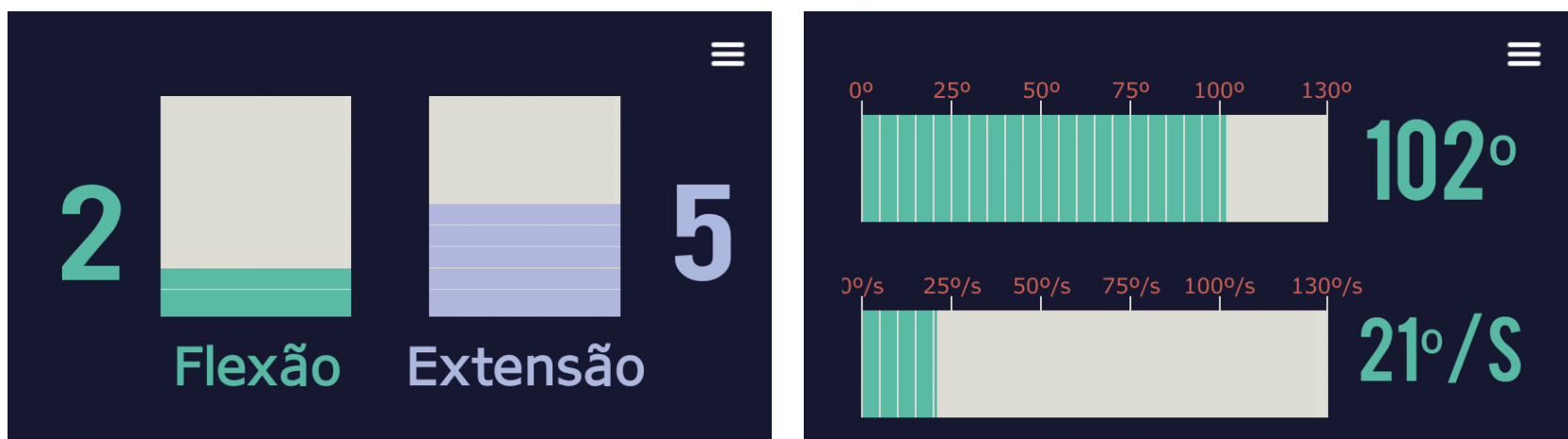

Figure 1. Knowledge of performance mobile assistance. The mobile application can provide a physiologically based feedback (left panel), and a kinematic based feedback (right panel) based on muscular activation or movement kinematic data respectively. 'Flexão' and 'Extensão' on the right panel is Portuguese for 'Flexion' and 'Extension' respectively.

stroke survivors with appropriate KP to assist and improve their rehabilitation process. The Android application has been developed with Unity 3D (Unity Technologies, San Francisco, USA) using C\# as the main programming language and Java for a custom Bluetooth JAR library through the Android SDK since Unity 3D does not allow direct access to the Bluetooth capabilities of Android devices. With this plug-in we were able to connect to the mPower 1000 device for bi-directional communication.

\subsubsection{Physiologically based Knowledge of \\ Performance}

In this KP mode, the mobile application presents feedback based on the physiological readings of the mPower 1000. Biceps and triceps EMG activation are represented in real-time as vertical bars accompanied by a numerical value (see figure 1, left panel) under the label "flexion" and "extension". The bar values correspond to normalized EMG activation values, with 0 and 10 corresponding to the minimum and maximum muscular activation levels, respectively. This view enables the user to see their muscular activation patterns easily represented as bars, thus allowing the user to better understand how successful movement results from muscle activation.

\subsubsection{Kinematic based Knowledge of Performance}

In this KP mode, the mobile application represents the real time position and velocity of the arm movements in degrees/s (see figure 1, right panel). Thus, this configuration relies on arm movement kinematic data and presents it, consistent with the physiologically based representation, as bars and their corresponding numerical value. Both kinematic and physiological data were chosen to be represented with the same amount of information channels (two bars) in a visually consistent manner. However, given the similarity of the two feedback representations and to avoid confusions, the kinematic based feedback is presented using horizontal bars.

\subsection{Data collection}

For further analysis, the system collects synchronously all kinematic and physiological data during each training session. Recorded data include pre-processed normalized amplitude values of the envelope of EMG signals from biceps and triceps $(0-14)$, position of the arm (degrees), time (ms), average speed (degrees/s) and the number of arm flexion's and extensions. The application logs all data directly to a file on the mobile device. Data are sampled every $100 \mathrm{~ms}$ and stored as a CSV text file.

\section{PILOT EVALUATION}

Three stroke survivors with an average age of 67 years participated in a pilot evaluation of the system (table 1). All patients were informed about the purpose of the study and signed an informed consent form prior to participation. Participants sat with the mPower 1000 robotic arm placed on their paretic arm. An Android tablet was presented in front of them with the application providing KP feedback (see figure 2).

All participants were exposed to the mPower device prior to the tests in order to learn how to use it. After the training period, patients were presented with the Android tablet with both KP feedback forms, that is, based on EMG activation and based on movement kinematics. The session consisted on the repetition of a simple arm movement (arm flexion and extension) during blocks of 4 minutes (see figure 3). Between blocks, patients had a few minutes to rest. After they completed the training, patients were

Table 1. Patient demographics

\begin{tabular}{|c|c|c|c|c|}
\hline Patient & Age & Stroke type & Side & $\begin{array}{c}\text { Time post- } \\
\text { stroke }\end{array}$ \\
\hline 1 & 74 & Ischemic & Right & 40 weeks \\
\hline 2 & 54 & Ischemic & Left & 5 weeks \\
\hline 3 & 78 & n/a & Right & 30 weeks \\
\hline
\end{tabular}

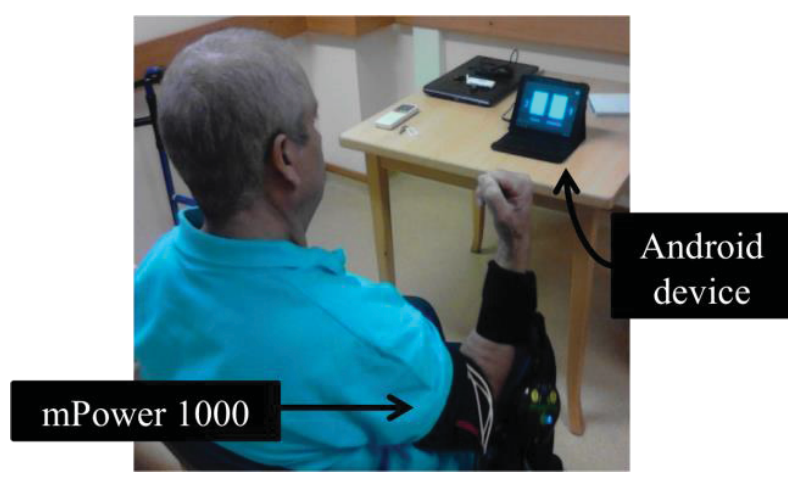

Figure 2. Stroke patient using the mobile knowledge of performance system for upper limb rehabilitation. 

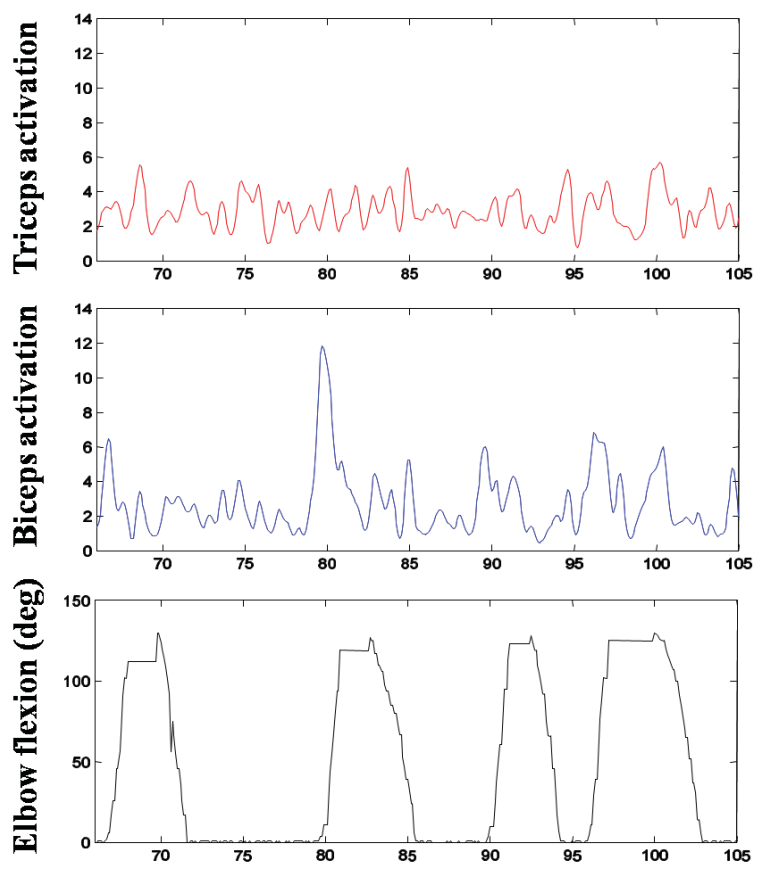

Figure 3. Data sample of one patient performing repetitive arm flexion and extension training using the mPower 1000 myo-electric orthosis.

asked about their opinion about each type of KP feedback.

All participants reported that they had some difficulty in understanding the physiologically based feedback. We believe that the large oscillations on the EMG signals combined with the need of understanding the antagonistic nature of biceps and triceps EMG for the generation of the correct movement, made the physiologically based feedback less intuitive. When asked about which mode they preferred, all participants favoured the more direct relation between kinematic feedback and movement execution, claiming that it was easier to understand.

\section{DISCUSSION AND CONCLUSIONS}

In this project, we developed a mobile system able to deliver realtime feedback on knowledge of performance based on kinematic or physiological information. Furthermore, we performed a pilot evaluation with stroke survivors to compare the two modes of feedback. Patients reported that the kinematic type of feedback was easier to understand. Unfortunately, we observed that also cognitive deficits derived from stroke interfered with the feedback comprehension, which resulted in a small sample of patients having criteria for participating in this evaluation. Despite these limitations, we believe that this tool has potential for supporting specific stroke survivors during their rehabilitation process. This mobile system does not only assist in action execution by virtue of the displayed feedback, contributing to generating knowledge of performance, it also serves to quantitatively assess and monitor changes in the muscular activation patterns of the biceps and triceps, making it possible to quantify long term changes and improvements (see figure 3). Future work includes conducting further experiments to better understand both the effect of the nature of the feedback provided for knowledge of performance as well as its long term implications in the recovery of normal arm kinematics and muscle activation patterns.

\section{ACKNOWLEDGEMENTS}

This work is supported by the European Commission through the RehabNet project - Neuroscience Based Interactive Systems for Motor Rehabilitation - EC (303891 RehabNet FP7-PEOPLE2011-CIG), and by the Fundação para a Ciência e Tecnologia (Portuguese Foundation for Science and Technology) through SFRH/BPD/84313/2012, SFRH/BD/97117/2013, and Projeto Estratégico - LA 9 - 2013-2014.

\section{REFERENCES}

[1] Feigin, V. L., S. Barker-Collo, et al. (2008). Long-term neuropsychological and functional outcomes in stroke survivors: current evidence and perspectives for new research. Int J Stroke 3(1): $33-40$.

[2] Legg, L. A., A. E. Drummond, et al. (2006). Occupational therapy for patients with problems in activities of daily living after stroke. Cochrane Database Syst Rev(4): CD003585.

[3] Dimyan, M. A. and L. G. Cohen (2011). Neuroplasticity in the context of motor rehabilitation after stroke. Nat Rev Neurol 7(2): 76-85.

[4] Bowden, M. G., M. L. Woodbury, et al. (2013). Promoting neuroplasticity and recovery after stroke: future directions for rehabilitation clinical trials. Curr Opin Neurol 26(1): 37-42.

[5] Kilduski, N. C., \& Rice, M. S. (2003). Qualitative and Quantitative Knowledge of Results: Effects on Motor Learning. American Journal of Occupational Therapy. Vol. 57 no. 3 329336.

[6] Bermudez i Badia, S., Morgade, A. G., Samaha, H., \& Verschure, P. F. M. J. (2013). Using a Hybrid Brain Computer Interface and Virtual Reality System to Monitor and Promote Cortical Reorganization through Motor Activity and Motor Imagery Training. IEEE Transactions on Neural Systems and Rehabilitation Engineering, 21(2), 174-181.

[7] Fasoli, S. E., Krebs, I. H., Stein, J., Frontera, W. R., Hughes, R., \& Hogan, N. (2004). Robotic Therapy for Chronic Motor Impairments After Stroke: Follow-up Results. Arch Phys Med Rehabil Vol 85 .

[8] Burke, J. W., McNeill, M. D., Charles, D. K., Morrow, P. J., Cr0osbie, J. H., \& McDonough, S. M. (2009). Serious Games for Upper Limb Rehabilitation Following Stroke. Conference in Games and Virtual Worlds for Serious Applications .

[9] Cameirão, M. S., Badia, B. S., Oller, D. E., \& Verschure, P. F.M. J. (2010). Neurorehabilitation using the virtual reality based Rehabilitation Gaming System: methodology, design, psychometrics, usability and validation. Journal of neuroengineering and rehabilitation, 7:48. 